\title{
VARIATIONAL IRREVERSIBLE THERMODYNAMICS OF HEAT AND MASS TRANSFER IN POROUS SOLIDS: NEW CONCEPTS AND METHODS*
}

\author{
BY \\ M. A. BIOT \\ Royal Academy' of Belgium
}

\begin{abstract}
A recently developed variational principle of virtual dissipation along with a new approach to the thermodynamics of open systems is applied to coupled mass and heat transfer in a porous solid containing a fluid. General differential field equations are derived directly from the variational principle. A general energy flux theorem is formulated. Vapor-liquid phase transition and capillary condensation are discussed. Field equations for nonequilibrium adsorption are also obtained. Lagrangian equations with generalized coordinates are derived directly from the variational principle without use of the field equations. They provide the foundation of finite-element methods as well as of many other techniques particularly suitable in geothermal systems analysis.
\end{abstract}

1. Introduction. A principle of virtual dissipation has recently been developed generalizing d'Alembert's principle to nonlinear dissipative thermodynamic systems [1]. This new principle is a natural outgrowth of earlier work providing a variational-Lagrangian formulation of linear thermodynamics [2]. Application of this variational principle provides directly both the field equations of general continuous systems as well as the corresponding Lagrangian equations with generalized coordinates. The Lagrangian equations thus obtained constitute a powerful tool for systems analysis of very complex physical and technological systems. They are formulated from basic physical invariants of the system without a priori detailed knowledge of the field differential equations. This is in contrast with current procedures which derive variational principles from the particular differential field equations for each type of problem.

An important aspect of the new approach is its unified interdisciplinary nature, which embodies a complete synthesis between mechanics and thermodynamics. This is well illustrated by its application to the nonlinear thermorheology of continua [3] which covers a large category of phenomena.

Another innovation which has considerably enlarged the field of application of the variational technique is the development of an entirely new fundamental approach to the thermodynamics of open systems $[4,5,6]$. The concept of "thermobaric potential" replaces Gibbs' chemical potential and bypasses the well-known difficulties associated with it. A nother essential concept which has been introduced is that of entropy convection which is given a precise definition. This has led to new results in the theory of chemical reactions $[4,5]$. Along with the principle of virtual dissipation, these new concepts were applied to the non-isothermal dynamics of Newtonian and non-Newtonian fluids [6].

\footnotetext{
* Received September 30, 1977.
} 
A nother application is to the non-isothermal finite strain mechanics of porous solids with a fluid mixture and nonporous solids with thermomolecular diffusion of solutes [7].

The present treatment is an application to heat and mass transfer in a rigid porous medium containing a single fluid. While the context is more restricted than in a concurrent paper [7], the fluid mechanics of the pore fluid is analyzed here in considerably more detail. In particular, phase transitions and capillary condensation are considered as well as nonequilibrium adsorption effects. New theorems are also derived which govern the energy flux.

The results obtained earlier [7] may readily be combined with the present treatment to take into account solid deformation and coupled diffusion effects when the pores contain a fluid mixture.

2. New concepts in the thermodynamics of open systems. We shall start with a brief account of newly developed concepts and methods [4, 5, 6]. We consider a system constituted by a primary cell $C_{P}$, a large rigid supply cell $C_{S}$ and a large isothermal reservoir at a constant temperature $T_{0}$ called a thermal well $T W$. The cells $C_{P}$ and $C_{S}$ with the thermal well $T W$ constitute a "hypersystem". In any reversible transformation the work accomplished on the hypersystem defines the collective potential. No external matter or heat is added to the hypersystem. Matter and heat are transferred internally within the hypersystem by a reversible process which involves the combined use of heat engines and mechanical pumps.

In the particular case of a porous medium with a rigid matrix, we assume a primary cell of unit volume, containing a compressible viscous fluid in the pores. The fluid and the solid are assumed in thermal equilibrium at the temperature $T$. A large rigid supply cell contains the same fluid at the temperature $T_{0}$ and pressure $p_{0}$.

An important property of the collective potential $\mathcal{V}$ is the fact that it depends only on the state variables of the primary cell. This can easily be shown by noting that by definition the collective potential is the internal energy of the collective system. We may write

$$
\vartheta=\mathcal{U}+H_{0}
$$

where $\mathcal{U}$ is the collective internal energy of the pair of cells $C_{P}+C_{s}$ and $H_{0}$ is the thermal energy acquired by the thermal well. The quantities $v$ and $\mathcal{U}$ denote increases from a zero initial value. Since the transformation is reversible the total entropy change of the hypersystem is zero. Hence

$$
\mathcal{S}+\left(H_{0} / T_{0}\right)=0
$$

where $S$ is the collective entropy of the pair of cells $C_{P}+C_{S}$. Elimination of $H_{0}$ between Eq. (2.1) and (2.2) yields

$$
\mathcal{V}=\mathcal{U}-T_{0} \delta .
$$

The initial state of the primary cell for which we put $\mathcal{u}=\delta=\vartheta=0$ is chosen arbitrarily to suit the problem. The primary cell is considered to be jacketed and rigid while a mass $m$ of fluid may be added in the pores by injection through the jacket. The thermodynamic state of the primary cell is determined by its temperature $T$ and the mass $m$ of pore fluid added. On the other hand, the thermodynamic state of the large and rigid supply cell is determined by the same mass $m$ of fluid which had to be extracted. Hence $T$ and $m$, which define the state of the primary cell, also define the state of the collective system $C_{P}+C_{S}$. 
As a consequence $\mathcal{U}, \mathcal{S}$ as well as $\mathcal{V}$ are completely determined by $T$ and $m$. We have therefore referred to $\mho$ as the cell potential. The cell potential may of course be considered as a measure of availability. However, its use as a new thermodynamic potential different from the classical definitions was introduced by the author as a basic concept in irreversible thermodynamics [2] and referred to as a generalized free energy. In later years the term "exergy" was also used by some authors to designate related concepts.

We now consider the transfer of a mass of fluid $d m$ from the supply cell $C_{S}$ to the primary cell $C_{P}$. This transfer is accomplished reversibly by the combined use of mechanical pumps and heat pumps and has been called a thermobaric transfer $[4,5]$. The work accomplished is written

$$
d V=\psi d m .
$$

The differential coefficient $\psi$ is the thermobaric potential, $[4,5]$. Its value is

$$
\psi(p, T)=\int_{p_{0} T_{0}}^{p T}\left(\frac{d p}{\rho}+\theta d \bar{s}\right)
$$

where

$$
\theta=T-T_{0} .
$$

The integration is along an arbitrary path of continuous variation of pressure $\rho$, temperature $T$ and specific mass $\rho$, and $d \bar{s}$ is the entropy differential of the unit mass along this path. The notation $p$ in the upper limit of the integral denotes the pressure of an external fluid in thermodynamic equilibrium with the pore fluid. Hence it is defined thermodynamically and is not necessarily the fluid pressure in the pores which in some cases cannot be clearly defined. For simplicity we have used the same notation $p$ and $T$ as variables along the path of integration and for their values at the upper limit of integration. Expression (2.5) was derived and discussed earlier [4,5]. It represents the reversible work necessary to extract a unit mass at the pressure $p_{0}$ from the supply cell, bring it to a pressure $p$ and temperature $T$ in equilibrium with the primary cell and inject it adiabatically into it. This continuous process is accomplished reversibly by the simultaneous use of mechanical pumps and heat pumps. The first term in expression (2.5) represents the work of the mechanical pumps and the second term the work of the heat pumps operating between the temperatures $T$ and $T_{0}$.

Note that for an isothermal system with $\theta=0$ expression (2.5) reduces to the "pressure function"

$$
\psi=\int_{p_{0}}^{p} \frac{d p}{\rho}
$$

of classical fluid mechanics.

In addition to adding masses to the primary cell, we may also add heat directly by means of a heat pump. With the appropriate additional term, expression (2.4) for the increase of cell potential becomes

$$
d V=\psi d m+\theta d s_{t}
$$

where $\theta d s_{T}$ is the work of the heat pump required between the temperatures $T$ and $T_{0}$ in order to inject reversibly an amount of heat energy $T d s_{T}$. The differential $d s_{T}$ is the increase of entropy of the primary cell due to this heat injection. This quantity is not a state variable. Actually, one of the state variables is the collective entropy $S$ of the pair of 
cells $C_{P}+C_{S}$. Its differential is

$$
d S=\bar{s} d m+d s_{T}
$$

where

$$
\bar{s}(p, T)=\int_{p_{0} T_{0}}^{p T} d \dot{s}
$$

is the relative specific entropy of the fluid. Relation (2.9) derived earlier $[4,5]$ is obtained by considering the collective entropy increase of the system $C_{p}+C_{s}$ including the mass $d m$ when the latter is brought to equilibrium with the primary cell. This entropy increase is $\bar{s}$ $d m$. During reversible injection into $C_{p}$ the total entropy does not change; hence $\bar{s} d m$ represents the collective entropy increase of $C_{p}+C_{s}$ after addition of the mass $d m$ to the primary cell $C_{p}$. Therefore $\bar{s} d m$ is the increase of $\delta$ due to convection, while $d s_{T}$ is the increase due to the direct addition of heat energy by conduction.

Elimination of $d s_{T}$ between Eqs. (2.8) and (2.9) yields

$$
d \mathrm{U}=\Phi d m+\theta d s
$$

where

$$
\Phi=\psi-\theta \bar{s}
$$

is the convective potential $[4,5]$. The differentials in $(2.11)$ are now state variables. It is now valid for irreversible transformations while (2.9) is not. We derive with $m$ and $\&$ as independent variables,

$$
\partial \vartheta / \partial m=\Phi, \quad \partial V / \partial S=\theta .
$$

It is interesting to substitute the value (2.11) of $d 0$ into Eq. (2.3). We find

$$
d^{\mathfrak{U}}=\Phi d m+T d S .
$$

This is formally similar to the expression used by Gibbs [8] to define the chemical potential $\mu$ as the differential coefficient of $d m$. However, there are several fundamental differences. First, $\mathcal{U}$ and $S$ are the collective internal energy and collective entropy of the pair of cells $C_{p}+C_{s}$. Second, $\Phi$ is not defined by Eq. (2.14) as the differential coefficient of $d m$ but independently by Eq. (2.12). Hence relation (2.14) constitutes a theorem. Third, $d S$ is defined in a precise way by (2.9) as the sum of convective and conductive terms. Finally, within a given hypersystem the variables in (2.14) do not involve any undetermined constants.

The convective potential $\Phi$ may also be written in a different form by introducing into Eq. (2.12) the values (2.5) and (2.10) for $\psi$ and $\bar{s}$. We derive

$$
\Phi=\bar{\epsilon}-T \bar{s}
$$

where

$$
\bar{\epsilon}=\int_{p_{0} T_{0}}^{p T}\left(\frac{d p}{\rho}+T d \bar{s}\right)
$$

is the increase of collective internal energy of the pair of cells $C_{p}+C_{s}$ in the thermobaric transfer of a unit mass from $C_{s}$ to $C_{p}$. We may also write

$$
\bar{\epsilon}=\frac{p}{\rho}-\frac{p_{0}}{\rho_{0}}+\bar{u}
$$


where

$$
\bar{u}=\int_{p_{0} T_{0}}^{p T}\left[-p d\left(\frac{1}{\rho}\right)+T d \bar{s}\right] .
$$

Note that this integral represents the classical increase of the fluid internal energy per unit mass when its pressure and temperature vary from $p_{0}, T_{0}$ to $p, T$. Hence $\bar{\epsilon}$ may be interpreted as the increase of specific enthalpy of the fluid when the pressure and temperature vary from $p_{0}, T_{0}$ to $p, T$. In analogy with $\bar{s}$, which was referred to as the relative specific entropy, we may call $\bar{\epsilon}$ the relative specific enthalpy of the fluid.

In contrast with classical concepts and procedures, the quantities $\Phi, \bar{s}$ and $\bar{\epsilon}$ are completely defined for a given hypersystem and do not involve any undetermined constants.

3. Relations between state variables. Liquid-vapor transitions. In Eq. (2.11) for $d V$ we shall consider $m$ and $S$ as state variables of the primary cell of unit volume of porous medium. We recall that $m$ is the mass of pore fluid added to the primary cell starting from an arbitrary initial state, of temperature $T^{\prime}$ and pore fluid pressure $p^{\prime}$. As already pointed out, the collective quantities $\mathcal{U}, \delta$, and $\mathcal{V}$ for the primary cell are put equal to zero in this initial state while $m$ is initially zero by definition.

The two state variables $m$ and $\mathcal{S}$ are related to other state variables such as the temperature $T$, the pore pressure $p$, etc. We shall now examine in more detail such relationships. Note that we have defined the pore pressure $p$ as that of the fluid located outside the cell and in thermodynamic equilibrium with the pore fluid. This takes care of all kinds of physical-chemical and capillary interactions in the pores.

However, it is very instructive to consider the case where the interaction of fluid and solid is negligible. In this case it makes no difference if we consider the fluid to be located outside the solid. The pressure $p$, density $\rho$, and temperature $T$ of the fluid obey the equation of state

$$
p=p(\rho, T) .
$$

Its relative specific entropy, defined by Eq. (2.10), is

$$
\bar{s}(\rho, T)=\int_{p_{0} T_{0}}^{p T} d \bar{s} .
$$

The volume occupied by the fluid in the pores is

$$
f=\frac{m_{0}+m}{\rho},
$$

where $m_{0}$ is the initial mass in the pores and $f$ is the porosity, i.e. the pore volume per unit volume of porous medium. We may express $p$ and $\bar{s}$ as functions of $m$ and $T$ :

$$
p=p\left(\frac{m_{0}+m}{f}, T\right), \quad \bar{s}=\bar{s}\left(\frac{m_{0}+m}{f}, T\right) .
$$

Since the fluid is located outside the solid, the collective entropy $\delta$ may be evaluated separately for the fluid and the solid and added together. The mass $m_{0}$ of fluid initially at the temperature $T^{\prime}$ and density $\rho^{\prime}=m_{0} / f$ is brought to the temperature $T$ and density $\rho=$ $\left(m_{0}+m\right) / f$. Its collective entropy increases by the most 


$$
m_{0}\left[\bar{s}\left(\frac{m_{0}+m}{f}, T\right]-\bar{s}\left(\frac{m_{0}}{f}, T^{\prime}\right)\right] .
$$

A nother mass $m$ of fluid is extracted reversibly from the supply cell and brought to the density $\rho$ and temperature $T$. Its collective entropy increases by the amount

$$
m \bar{s}\left(\frac{m_{0}+m}{f}, T\right) \text {. }
$$

The solid matrix is brought from the temperature $T$ to $T$ with an entropy increase

$$
\oint_{s}=m_{s} \int_{T^{\prime}}^{T} \frac{c_{s}(T)}{T} d T
$$

where $m_{s}$ is the mass of solid per unit volume of porous medium and $c_{s}(T)$ its specific heat as a function of the temperature. The total collective entropy of the primary cell is the sum of the three terms (3.5) (3.6) and (3.7). We write

$$
\mathcal{S}(m, T)=\left(m_{0}+m\right) \dot{s}\left(\frac{m_{0}+m}{f}, T\right)-m_{0} s\left(\frac{m_{0}}{f}, T\right)+S_{s} .
$$

This also provides the temperature $T$ as a function of $m$ and $S$ :

$$
T=T(m, \delta) \text {. }
$$

This relation may be represented in an entropy diagram, where $T$ is plotted as a function of $\mathcal{S}$ yielding a family of curves defined by $m$ as a parameter. In the region where liquid and vapor coexist, it also provides the saturation variable $\mathscr{X}$ representing the fraction of fluid mass in the vapor phase. Its value is $x=0$ for a pure liquid phase and $x$ $=1$ for a pure vapor phase.

Attention is called to the fact that $S$ is a better state variable than $T$ since in the wet vapor region the temperature does not provide any information on the amount of saturation described by the variable $x$.

Assuming again no interaction between fluid and solid, we use the same reasoning to evaluate the collective internal energy $\mathcal{U}$. We find

$$
\mathcal{u}(m, T)=\left(m+m_{0}\right) \bar{u}\left(\frac{m+m_{0}}{f}, T\right)-m_{0} \bar{u}\left(\frac{m_{0}}{f}, T^{\prime}\right)-m \frac{p_{0}}{\rho_{0}}+\mathcal{u}_{s}
$$

where

$$
\mathcal{u}_{s}=m_{s} \int_{T^{\prime}}^{T} c_{s} d T
$$

and $\bar{u}$ is defined by $(2.18)$ as

$$
\bar{u}=\int_{\rho_{0} T_{0}}^{\rho T}\left[-p d\left(\frac{1}{\rho}\right)+T d \bar{s}\right] .
$$

The term $-m p_{0} / \rho_{0}$ represents the negative work of extraction of the mass $m$ from the supply cell.

Since $T$ is a function of $m$ and $S$ by relation (3.10), we express

$$
\mathcal{u}=\mathcal{U}(m, s)
$$

as a function of $m$ and $\delta$. 
With this value for $\mathcal{U}$, the collective potential $(2.3)$ is

$$
\mathcal{V}(m, \S)=\mathcal{U}(m, \S)-T_{0} \S .
$$

It is interesting to verify expression (2.11) from this result by forming the differential

$$
d \mathcal{U}=d^{d} \mathcal{U}-T_{0} d S .
$$

From (3.11) we obtain

$$
d^{\alpha} \mathcal{U}=\left(\bar{u}-\frac{p_{0}}{\rho_{0}}\right) d m+\left(m+m_{0}\right) d \bar{u}+d \mathcal{U}_{s} .
$$

Since the pore volume $f$ is constant, Eq. (3.3) yields

$$
-\left(m+m_{0}\right) d\left(\frac{1}{\rho}\right)=\frac{d m}{\rho} .
$$

From this relation and Eq. (3.13) we derive

$$
\left(m+m_{0}\right) d \bar{u}=\frac{p}{\rho} d m+\left(m+m_{0}\right) T d \bar{s} .
$$

With this value, expression (3.17) becomes

$$
d \mathfrak{U}=\bar{\epsilon} d m+\left(m+m_{0}\right) T d \bar{s}+d \mathcal{U}_{s}
$$

where $\bar{\epsilon}$ is the relative specific fluid enthalpy defined by (2.17).

Since $d \mathfrak{u}_{s}=T d S_{s}$ the differential of (3.8) is

$$
d S=\bar{s} d m+\left(m+m_{0}\right) d \bar{s}+\frac{1}{T} d \mathcal{U}_{s} .
$$

From relations (3.20) and (3.21) we derive

$$
d \mathfrak{u}=(\bar{\epsilon}-T \bar{s}) d m+T d S
$$

and with this value of $d^{\mathfrak{U}} \mathrm{U}$, expression (3.16) becomes

$$
d \mathrm{U}=(\bar{\epsilon}-T \bar{s}) d m+\theta d S .
$$

This coincides with Eq. (2.11) by introducing the value (2.15) of $\Phi$.

In the more general case where fluid and solid interaction may not be neglected, the values of $\delta$ and $U$ must be obtained from more elaborate physical-chemical theories or from direct physical measurement. In the latter case we integrate expressions (2.9) and (2.14), assuming a reversible process:

$$
\mathcal{S}(m, T)=\int_{0 T^{\prime}}^{m T}\left(\bar{s} d m+d s_{T}\right)
$$

and

$$
\mathcal{U}(m, T)=\int_{0 T^{\prime}}^{m T}\left(\Phi d m+T d s_{T}\right) .
$$

In these integrals the values of $\bar{s}$ and $\Phi$ are measured physically as a function of $m$ and $T$. This requires the measurement as a function of $m$ and $T$ of the pore pressure $p$ which, according to our definition, is the pressure of a fluid in equilibrium with the pore fluid and 
located outside the pores. For example, because of capillary effect the fluid may be in liquid form in the pores while in equilibrium with its vapor outside at a higher pressure (see below). This outside pressure and the temperature determine the values of $\bar{s}$ and $\Phi$. In this more general case we may also determine an entropy diagram as described above.

4. Mass balance, entropy balance, entropy production and flux coupling. The porous medium is considered as a continuum. The rate of mass flow of the pore fluid per unit area of the medium is $M_{i}$, where the dot denotes a time derivative. Conservation of mass is expressed by

$$
\dot{m}=-\left(\partial \dot{M}_{i} / \partial x_{i}\right)
$$

where $x_{i}$ are cartesian coordinates and $\dot{m}$ is the rate of increase of fluid mass per unit volume. Integrating (4.1) with respect to time with zero initial values yields

$$
m=-\left(\partial M_{i} / \partial x_{i}\right) \text {. }
$$

In this relation $m$ is the increase of fluid mass per unit volume, and $M_{i}$, called the mass displacement $[6,9]$, is the total mass of fluid which has been flowing per unit area since the initial time $t=0$. Relation (4.2) constitutes a holonomic mass balance constraint.

In order to express the entropy balance we consider the rate of flow of heat energy per unit area $H_{i}$. The rate of increase of entropy per unit volume due to $\dot{H}_{i}$ is

$$
\dot{s}_{T}=-\frac{1}{T} \frac{\partial \dot{H}_{i}}{\partial x_{i}} \text {. }
$$

In order to take into account the entropy convected we consider a domain $\Omega$ of the continuum of boundary $A$. The rate of entropy increase of the whole domain $\Omega$ is

$$
\dot{S}_{\Omega}=\int_{\Omega} \dot{s}_{T} d \Omega-\int_{A} \bar{s} \dot{M}_{i} n_{i} d A .
$$

The second integral is the entropy convected into $\Omega$ through the boundary $A$, of unit normal $n_{i}$, and $\bar{s}$ is the relative specific entropy of the fluid at the boundary. This convection term provides the key to the treatment of open systems. It is derived by the same reasoning as used in the derivation of Eq. (2.9) for the case of a reversible transformation. With the value $(4.3)$ for $\dot{s}_{T}$ integration by parts yields

where

$$
\dot{S}_{\Omega}=\int_{\Omega} \dot{s}_{T} * d \Omega-\int_{A} \dot{S}_{i} n_{i} d A
$$

$$
\dot{s}_{T}^{*}=-\left(\dot{H}_{i} / T^{2}\right)\left(\partial T / \partial x_{i}\right)
$$

and

$$
\dot{S}_{i}=\dot{s} \dot{M}_{i}+\left(\dot{H}_{i} / T\right)
$$

The quantity $\dot{s}_{T}{ }^{*}$ is the rate of entropy production per unit volume due to thermal conduction while $\dot{S}_{i}$ is the total rate of entropy flow. We put

$$
\dot{S}_{i}^{T}=\dot{H}_{i} / T \text {. }
$$

Hence

$$
\dot{S}_{i}=\bar{s} \dot{M}_{i}+\dot{S}_{i}{ }^{T}
$$


where $\bar{s} \dot{M}_{i}$ is the rate of entropy flow due to fluid convection and $\dot{S}_{i}{ }^{T}$ is rate of entropy flow due to thermal conduction in the bulk fluid-solid medium.

Eq. (4.5) may also be expressed as a volume integral

$$
\dot{S}_{\Omega}=\int_{\Omega}\left(\dot{s}_{T} *-\frac{\partial \dot{S}_{i}}{\partial x_{i}}\right) d \Omega \quad ;
$$

hence we obtain the entropy balance equation $[6,7]$

$$
\dot{S}=-\frac{\partial \dot{S}_{i}}{\partial x_{i}}+\dot{s}_{T}^{*}
$$

where $S$ is the entropy per unit volume. It generalizes Meixner's relation [10] since it includes the entropy convection term $\dot{s} \dot{M}_{i}$ of Eq. (4.9). Putting

$$
\dot{s}=-\left(\partial \dot{S}_{i} / \partial x_{i}\right)
$$

and integrating with respect to time with zero initial values yields

$$
s=\left(\partial S_{i} / \partial x_{i}\right)
$$

where $s$ is the entropy supplied per unit volume by convection and conduction and $S_{i}$ is the total entropy displacement vector. This concept was introduced earlier in several publications [1, 3, 6, 9].

Note that Eq. (4.13) for the supplied entropy balance is analogous to (4.2) for mass balance and is also holonomic. This property plays an important role in the variational theory.

In the most general case of anisotropy the law of thermal conduction is

$$
\partial T / \partial x_{i}=-\lambda_{i j} \dot{H}_{j}
$$

where $\lambda_{i j}=\lambda_{j i}$ is the symmetric thermal local resistivity tensor of the bulk fluid-solid medium. Its symmetry is a consequence of Onsager's principle [11, 12]. It is a function

$$
\lambda_{i j}=\lambda_{i j}\left(m, T, x_{k}\right)
$$

of the fluid mass increase $m$ in the pores, the local temperature $T$ and also of the local coordinates $x_{k}$ if the bulk medium is not homogeneous. With the values (4.8) and (4.14) Eq. (4.6) becomes

$$
\dot{S}_{T}^{*}=\lambda_{i j} \dot{S}_{i}^{T} \dot{S}_{j}^{T}
$$

which yields the local rate of entropy production as a quadratic form in $\dot{S}_{i}{ }^{T}$ with coefficients depending on $m, T$ and $x_{i}$.

Until now we have assumed that the local rate of entropy production is due only to thermal conduction. Actually additional entropy production is due to the fluid flow rate. The rate of entropy production $\dot{s}^{*}$ in this case is expressed by a straightforward generalization of Eq. (4.16) as a quadratic form in $\dot{M}_{i}$ and $\dot{S}_{i}{ }^{T}$. We write

$$
T \dot{s}^{*}=C_{i j}{ }^{T} \dot{M}_{i} \dot{M}_{j}+2 C_{i j}{ }^{T M} \dot{M}_{i} \dot{S}_{j}{ }^{T}+T \lambda_{i j} \dot{S}_{i}{ }^{T} \dot{S}_{j}{ }^{T} .
$$

In the variational theory it is more convenient to consider the rate of dissipation $T \dot{s}^{*}$ instead of $\dot{s}^{*}[1,3,6]$. The coefficients are functions of $m, T$ and location $x_{i}$. The quadratic form embodies the local validity of Onsager's principle [11, 12] and symmetry properties

$$
C_{i j}{ }^{T}=C_{j i}{ }^{T}, \lambda_{i j}=\lambda_{j i} \text {. }
$$


The cross-term coefficient $C_{i j}{ }^{T M}$ represents the coupling between mass flow and heat flow as exemplified by the Knudsen effect [13].

With the total entropy production as expressed by (4.17) Eq. (4.11) retains the same form:

$$
\dot{S}=-\left(\partial \dot{S}_{i} / \partial x_{i}\right)+\dot{s}^{*} .
$$

Integration with respect to time with zero initial values yields

$$
\delta=-\left(\partial S_{i} / \partial x_{i}\right)+s^{*}=s+s^{*} .
$$

This is the integrated form of the entropy balance equation.

The thermodynamic state of the porous domain $\Omega$ is completely determined by the scalar fields $m$ and $\delta$. Eqs. (4.2) and (4.20) show that these state variables may be replaced by the two vector fields $S_{i}$ and $M_{i}$ and an additional scalar s*.

The rate of dissipation (4.17) may be written in terms of the variables $\dot{S}_{i}$ instead of $\dot{S}_{i}{ }^{T}$. From (4.9) we obtain

$$
\dot{S}_{i}{ }^{T}=\dot{S}_{i}-\dot{s} \dot{M}_{i} .
$$

Substitution of this value into (4.17) yields for the rate of dissipation

$$
T \dot{s}^{*}=C_{i j} \dot{M}_{i} \dot{M}_{j}+2 C_{i j}{ }^{M} \dot{M}_{i} \dot{S}_{j}+T \lambda_{i j} \dot{S}_{i} \dot{S}_{j}
$$

where

$$
\begin{gathered}
C_{i j}=C_{i j}{ }^{T}-\left(C_{i j}{ }^{T M}+C_{j i}{ }^{T M}\right) \bar{s}+T \lambda_{i j} \bar{s}^{2}, \\
C_{i j}{ }^{M}=C_{i j}{ }^{T M}-T \lambda_{i j} \bar{s} .
\end{gathered}
$$

We note that if $C_{i j}{ }^{T M}=0$ the coupling between $\dot{M}_{i}$ and $\dot{S}_{i}{ }^{T}$ vanishes; however, there remains a coupling term

$$
C_{i j}{ }^{M}=-T \lambda_{i j} \dot{s}
$$

between $\dot{M}_{i}$ and $\dot{S}_{i}$ whose nature is purely convective.

5. Variational principle of virtual dissipation. We consider the hypersystem constituted by the porous domain $\Omega$ the supply cell $C_{s}$ and the thermal well $T W$. An important property of the collective potential is its additivity. Hence we may write the collective potential of the domain $\Omega$ as

$$
V=\int_{\Omega} v d \Omega
$$

where $V d \Omega$ is the potential of the infinitesimal cells composing the domain, and $V$ is its local value per unit volume. Also

$$
\vartheta=\mathcal{U}-T_{0} \delta
$$

where $\mathcal{U}$ and $\delta$ are respectively the local values per unit volume of the collective internal energy and entropy of the cell, as defined in Sec. 2, in terms of the supply cell $C_{s}$.

In Sec. 2 the potential $\mathcal{V}$ is defined by means of reversible work on the hypersystem. We shall now consider the completely general case of an irreversible transformation. The first principle of thermodynamics applied to the system constituted by the domain $\Omega$ and the 
supply cell $C_{s}$ is written in variational form as

$$
\int_{\Omega} \delta u d \Omega=\delta W-I_{i} \delta q_{i} .
$$

The left-hand side is the increase of collective internal energy of the system $\Omega+C_{s}$. On the right-hand side we have the virtual work $\delta W$ of the external forces applied to $\Omega$ and the virtual work $-I_{i} \delta q_{i}$ of the reversed inertia forces in the sense of d'Alembert's principle. We denote by $I_{i}$ the generalized inertia forces associated with the variable $q_{i}$. Elimination of $u$ between Eqs. (5.2) and (5.3) yields

$$
\int_{\Omega}\left(\delta \mathcal{V}+T_{0} \delta \delta\right)=\delta W-I_{i} \delta q_{i} .
$$

Variations are applied only inside $\Omega$. Hence the variation of total entropy produced in $\Omega+$ $C_{s}$ is obviously

$$
\int_{\Omega} \delta S d \Omega=\int_{\Omega} s^{*} d \Omega
$$

where $\delta s^{*}$ is the variation of entropy produced locally per unit volume. Hence (5.4) becomes

$$
\int_{\Omega}\left(\delta V+T_{0} \delta s^{*}\right) d \Omega=\delta W-I_{i} \delta q_{i} .
$$

Furthermore, using the value (2.11) and the value (4.20) of $\mathcal{S}$, we derive

$$
\delta \mathcal{V}+T_{0} \delta s^{*}=\delta_{R} \tau+T \delta s^{*}
$$

where

$$
\delta_{R} \mathcal{V}=\Phi \delta m+\theta \delta s=\frac{\partial \mathcal{V}}{\partial m} \delta m+\frac{\partial \mathcal{V}}{\partial \delta} \delta s
$$

and $\delta_{R}$ denotes a restricted variation obtained by varying only $m$ and $s$, hence dropping $\delta s^{*}$ in the variation of $v$. Relation (5.6) becomes

$$
I_{i} \delta q_{i}+\int_{\Omega}\left(\delta_{R} \mho+T \delta s^{*}\right) d \Omega=\delta W .
$$

This constitutes the principle of virtual dissipation as derived earlier in a more general context $[1,3]$. In most problems the work of the external forces may be separated into two terms

$$
\delta W=-\delta G+\delta W^{\prime}
$$

where $\delta G$ is the virtual work of body forces derived from a potential field such is gravity, while $\delta W^{\prime}$ is the virtual work of other forces. We put

$$
\rho=G+\int_{\Omega} \vartheta d \Omega .
$$

This quantity is the mixed collective potential defined earlier [1,2] as a unified concept for the combined mechanics and thermodynamics of the system. By introducing the values 
(5.10) and (5.11) the principle of virtual dissipation becomes

$$
I_{i} \delta q_{i}+\delta_{R} \odot+\int_{\Omega} T \delta s^{*} d \Omega=\delta W^{\prime} .
$$

The integrand $T \delta s^{*}$ is the virtual dissipation per unit volume.

We have introduced [1,3] a local dissipation function which according to (4.22) is expressed as

$$
D=\frac{1}{2} T \dot{S}^{*}=\frac{1}{2} C_{i j} \dot{M}_{i} \dot{M}_{j}+C_{i j}{ }^{M} \dot{M}_{i} \dot{S}_{j}+\frac{1}{2} T \lambda_{i j} \dot{S}_{i} \dot{S}_{j}
$$

By Euler's theorem on homogeneous functions the local rate of dissipation may be written

$$
2 \mathfrak{D}=T \dot{s}^{*}=\frac{\partial \mathfrak{D}}{\partial \dot{M}_{i}} \dot{M}_{i}+\frac{\partial \mathcal{D}}{\partial \dot{S}_{i}} \dot{S}_{i}
$$

Hence the virtual dissipation due to variations $\delta M_{i}$ and $\delta S_{i}$ is

$$
T \delta s^{*}=\frac{\partial \mathcal{D}}{\partial \dot{M}_{i}} \delta M_{i}+\frac{\partial \mathcal{D}}{\partial \dot{S}_{i}} \delta S_{i} .
$$

By definition the values of the dissipative forces $\partial D / \partial \dot{M}_{i}$ and $\partial D / \partial \dot{S}_{i}$ remain frozen during the variations $[1,3]$.

6. Variational derivation of the field equations of mass and heat transfer. The mechanical and thermodynamic state of the porous domain $\Omega$ is completely described by three fields. They are the vector field $S_{i}$ of entropy displacement, the vector field $M_{i}$ of pore fluid mass displacement and the scalar field $s^{*}$ of entropy produced per unit volume.

We may vary these field variables arbitrarily inside the domain with zero variations at the boundary. The virtual work of the inertia forces is

$$
I_{i} \delta q_{i}=\int_{\Omega}\left(m_{0}+m\right) a_{i} \delta u_{i} d \Omega
$$

where $a_{i}$ is the fluid acceleration, $\delta u_{i}$ its virtual displacement and $m_{0}+m$ is the mass of fluid per unit volume, $m_{0}$ being the initial value. Since

$$
\left(m_{0}+m\right) \delta u_{i}=\delta M_{i}
$$

the variation $(6.1)$ becomes

$$
I_{i} \delta q_{i}=\int_{\Omega} a_{i} \delta M_{i} d \Omega
$$

If we denote by $S\left(x_{i}\right)$ the body force potential per unit mass, we may write

$$
G=\int_{\Omega}\left(m+m_{0}\right) \zeta d \Omega
$$

and

$$
\delta G=\int_{\Omega} \mathcal{S} \delta m d \Omega
$$

From (5.8), (5.11) and (6.5) we derive

$$
\delta_{R} \odot=\int_{\Omega}(\varphi \delta m+\theta \delta s) d \Omega
$$


where

$$
\varphi=\Phi+\mathcal{G}
$$

is a mixed convective potential.

For the isothermal case $\theta=0$ we obtain $\Phi=\psi$ where $\psi$ is expressed by (2.7). The potential (6.7) then coincides with the expression obtained by Hubbert in his formulation of Darcy's law [14].

We now introduce the values (5.15), (6.3) and (6.6) of the variations into the variational principle $(5.12)$ with $\delta W^{\prime}=0$. This yields

$$
\int_{\Omega}\left(a_{i} \delta M_{i}+\varphi \delta m+\theta \delta s+\frac{\partial D}{\partial \dot{M}_{i}} \delta M_{i}+\frac{\partial D}{\partial \dot{S}_{i}} \delta S_{i}\right) d \Omega=0
$$

Using the holonomic constraints (4.2) and (4.13) we write

$$
\delta m=-\frac{\partial}{\partial x_{i}} \delta M_{i} \quad \delta s=-\frac{\partial}{\partial x_{i}} \delta S_{i}
$$

We introduce these values into (6.8) and integrate by parts. Variations $\delta S_{i}$ and $\delta M_{i}$ are arbitrary and are chosen to vanish at the boundary. We therefore equate to zero the coefficients of these variations in the integrand and obtain

$$
a_{i}+\frac{\partial \varphi}{\partial x_{i}}+\frac{\partial D}{\partial \dot{M}_{i}}=0, \quad \frac{\partial \theta}{\partial x_{i}}+\frac{\partial D}{\partial \dot{S}_{i}}=0
$$

To these six equations we add a seventh equation (5.14), i.e.

$$
T \dot{s}^{*}=2 D \text {. }
$$

Together they govern the seven field components $M_{i}, S_{i}$ and $s^{*}$.

The acceleration of the fluid is

$$
a_{i}=\frac{\partial v_{i}}{\partial t}+v_{j} \frac{\partial v_{i}}{\partial x_{j}}
$$

where the average fluid velocity is

$$
v_{i}=\dot{M}_{i} /\left(m_{0}+m\right) \text {. }
$$

The acceleration may be written in several well-known alternative forms. For example, multiplying eq. (6.12) by $m_{0}+m$ and taking into account the condition of mass conservation

$$
\partial m / \partial t=-\left(\partial \dot{M}_{i} / \partial x_{i}\right)
$$

we obtain

$$
\left(m_{0}+m\right) a_{i}=\frac{\partial \dot{M}_{i}}{\partial t}+\frac{\partial}{\partial x_{j}}\left(\frac{\dot{M}_{i} \dot{M}_{j}}{m+m_{0}}\right)
$$

which brings out the change and flow of momentum.

Another form is obtained by introducing the vector

$$
Q=\mathbf{v} \times \operatorname{curl} \mathbf{v}
$$


where $\mathrm{v}$ is the velocity $v_{i}$. The acceleration is

$$
a_{i}=\frac{\partial v_{i}}{\partial t}+\frac{1}{2} \frac{\partial v^{2}}{\partial x_{i}}-\mathfrak{Q}_{i}
$$

where $Q_{i}$ are the components of $Q$ and $v^{2}=v_{i} v_{i}$. It is interesting to note that $Q_{i}=0$ in one-dimensional, axially symmetric, and spherically symmetric flows.

A nother form of the field equations (6.10) is obtained if we vary $S_{i}{ }^{T}$ instead of $S_{i}$. The virtual dissipation in this case is obtained by using a dissipation function $D^{T}$ derived from (4.17) and expressed in terms of $\dot{M}_{i}$ and $\dot{S}_{i}{ }^{T}$. We write

$$
\mathfrak{D}^{T}=\frac{1}{2} T \dot{S}^{*}=\frac{1}{2} C_{i j}{ }^{T} \dot{M}_{i} \dot{M}_{j}+C_{i j}{ }^{T M} \dot{M}_{i} \dot{S}_{j}{ }^{T}+\frac{1}{2} T \lambda_{i j} \dot{S}_{i}{ }^{T} \dot{S}_{j}{ }^{T}
$$

The virtual dissipation in this case becomes

$$
T \delta s^{*}=\frac{\partial \mathcal{D}^{T}}{\partial \dot{M}_{i}} \delta M_{i}+\frac{\partial \mathscr{D}^{T}}{\partial \dot{S}^{T}} \delta S_{i}{ }^{T}
$$

We substitute the value (6.19) for $T \delta s^{*}$ instead of (5.15) into the variational principle (5.12) and integrate by parts, taking into account the constraints (6.9) for $\delta m$ and $\delta s$. We obtain

$$
\int_{\Omega}\left(a_{i} \delta M_{i}+\frac{\partial \varphi}{\partial x_{i}} \delta M_{i}+\frac{\partial \theta}{\partial x_{i}} \delta S_{i}+\frac{\partial D^{T}}{\partial \dot{M}_{i}} \delta M_{i}+\frac{\partial \mathcal{D}}{\partial \dot{S}^{T}} \delta S_{i}{ }^{T}\right) d \Omega=0
$$

From (4.9) the variation of $\delta S_{i}$ is

$$
\delta S_{i}=\bar{s} \delta M_{i}+\delta S_{i}{ }^{T}
$$

We introduce this value into the variational principle (6.20), where $\delta M_{i}$ and $\delta S_{i}{ }^{T}$ are now arbitrary, and equate to zero the coefficients of these variations. Taking into account the values (2.5) and (6.7) of $\psi$ and $\varphi$ we derive

$$
a_{i}+\frac{1}{p} \frac{\partial p}{\partial x_{i}}+\frac{\partial S_{j}}{\partial x_{i}}+\frac{\partial D^{T}}{\partial \dot{M}_{i}}=0 \quad, \quad \frac{\partial \theta}{\partial x_{i}}+\frac{\partial D^{T}}{\partial \dot{S}_{i}{ }^{T}}=0 .
$$

This brings out explicitly the fluid pressure gradient in the field equations. For $\dot{M}_{i}=0$ the last equations express thermal conduction.

Capillary condensation. The field equations (6.10) are valid if the fluid is a vapor which condenses as a liquid as concave droplets on the walls of the pores. Because of local equilibrium the value $\varphi$ is the same whether referred to the vapor or the liquid. However, in this case the velocity and acceleration of the fluid are different for the vapor and the liquid. This may be taken into account by defining an average accleration $a_{i}$ such that $a_{i} \delta M_{i}$ represents the virtual work of the inertia forces. The field equations retain the form (6.10) because they involve only the total mass displacement of vapor and liquid. The effect of capillary condensation is embodied in the coefficients of the dissipation function which depend on and the fraction $X$ of vapor present. Hence according to the entropy diagram the coefficients may be expressed as functions of $m$ and $s$. It should be noted that in a more refined treatment a hysteresis effect should be introduced in these functions of $m$ and $\delta$ because condensation actually depends on the time history of the system.

In the case of capillary condensation the vapor pressure $p_{V}$ is different from the liquid pressure $p_{L}$. This is due to surface tension. However, $\Phi$ and hence also $\varphi=\Phi+\mathcal{G}$ are the same for both fluids since local equilibrium is assumed. The fluid pressures are easily evaluated by considering the convective potential $\Phi_{T}$ of the fluid at the pressure $p$ and 
temperature $T$ for a plane liquid-vapor interface. We may write

$$
\Phi-\Phi_{T}=\int_{p T}^{p_{v} T} \frac{d p_{V}}{\rho_{V}}=\int_{p T}^{p_{L} T} \frac{d p_{L}}{\rho_{L}}
$$

where $\rho_{V}$ and $\rho_{L}$ are respectively the vapor and liquid densities. If the pressure differences are small, Eq. (6.23) yields approximately

$$
\left(p_{V}-p\right) / \rho_{V}=\left(p_{L}-p\right) / \rho_{L}
$$

where $\rho$ and $\rho_{L}$ are vapor and liquid densities at $p$ and $T$.

The difference

$$
p_{V}-p_{L}=p_{c}
$$

is called the capillary pressure [15]. Solving Eqs. (6.24) and (6.25) for $p_{V}$ and $p_{L}$, we obtain

$$
\begin{gathered}
p_{V}=p-\frac{\rho_{V}}{\rho_{L}-\rho_{V}} p_{c} \cong p-\frac{\rho_{V}}{\rho_{L}} p_{c}, \\
p_{L}=p-\frac{\rho_{L}}{\rho_{L}-\rho_{V}} \cong p-p_{c} .
\end{gathered}
$$

When the condensed liquid presents concave surfaces, $p_{c}>0$. For example, if the concave surface is a hollow hemisphere of radius $r$,

$$
p_{c}=2 \gamma / r
$$

where $\gamma$ is the surface tension. Hence in this case

$$
p_{L}<p_{V}<p \text {. }
$$

7. Energy flux theorem. A very general and fundamental energy flux relation may be derived directly from the field equations (6.10). We add these field equations after multiplying the first three by $\dot{M}_{i}$ and the last three by $\dot{S}_{i}$. Taking into account expression (5.14) for $T \dot{s}^{*}$ and noting that $\partial \theta / \partial s_{i}=\partial T / \partial x_{i}$, we obtain

$$
\dot{M}_{i} a_{i}+\frac{\partial \varphi}{\partial x_{i}} \dot{M}_{i}+\frac{\partial T}{\partial x_{i}} \dot{S}+T \dot{s}^{*}=0 .
$$

By introducing the values (4.2), (4.13) from $m$ and $s$ and noting that according to (4.20) we may put $\dot{s}=\dot{s}+\dot{s}^{*}$, Eq. (7.1) becomes

$$
\dot{M}_{i} a_{i}+\frac{\partial}{\partial x_{i}}\left(\varphi \dot{M}_{i}+T \dot{S}_{i}\right)+\varphi \dot{m}+T \dot{S}=0 .
$$

The last terms may be expressed in the following form:

$$
\varphi \dot{m}+T \dot{S}=\Phi \dot{m}+\theta \dot{S}+g \dot{m}+T_{0} \dot{S} \quad .
$$

From relation (2.11) we derive

$$
\Phi_{\dot{m}}+\theta \dot{S}=\dot{v}
$$

and from $(2.3)$

$$
\dot{U}+T_{0} \dot{\delta}=\dot{U}^{0} .
$$


Hence Eq. (7.2) becomes

$$
\dot{M}_{i} a_{i}+\frac{\partial}{\partial x_{i}}\left(\varphi \dot{M}_{i}+T \dot{S}_{i}\right)+\dot{\mathrm{U}}+g \dot{m}=0 .
$$

The kinetic energy may be introduced in this equation by using the value (6.17) for $a_{i}$. Since the vectors $\dot{M}_{i}$ and $Q_{i}$ are perpendicular we have

$$
\dot{M}_{i} Q_{i}=0 \text {. }
$$

We derive

$$
\dot{M}_{i} a_{i}=\dot{M}_{i} \frac{\partial v_{i}}{\partial t}+\frac{1}{2} \dot{M}_{i} \frac{\partial v^{2}}{\partial x_{i}} .
$$

By definition (6.13) the rate of mass flow is

$$
\dot{M}_{i}=m^{\prime} v_{i}
$$

where $m^{\prime}=m+m_{0}$ is the total fluid mass per unit volume. We may write

$$
\begin{gathered}
\dot{M}_{i} \frac{\partial v_{i}}{\partial t}=\frac{1}{2} m^{\prime} v_{i} \frac{\partial v_{i}}{\partial t}=\frac{1}{2} \frac{\partial}{\partial t}\left(m^{\prime} v^{2}\right)-\frac{1}{2} v^{2} \frac{\partial m}{\partial t}, \\
\frac{1}{2} \dot{M}_{i} \frac{\partial v^{2}}{\partial x_{i}}=\frac{1}{2} \frac{\partial}{\partial x_{i}}\left(\dot{M}_{i} v^{2}\right)-\frac{1}{2} v^{2} \frac{\partial \dot{M}_{i}}{\partial x_{i}} .
\end{gathered}
$$

By taking into account the condition of conservation of mass (6.14), substitution of the values (7.10) into (7.8) yields

$$
\dot{M}_{i} a_{i}=\frac{1}{2} \frac{\partial}{\partial t}\left(m^{\prime} v^{2}\right)+\frac{1}{2} \frac{\partial}{\partial x_{i}}\left(\dot{M}_{i} v^{2}\right) .
$$

With this value Eq. (7.6) becomes

$$
\frac{\partial F_{i}}{\partial x_{i}}+\frac{1}{2} \frac{\partial}{\partial t}\left(m^{\prime} v^{2}\right)+\dot{U}+g \dot{m}=0
$$

where

$$
F_{i}=\varphi \dot{M}+T \dot{S}_{i}+\frac{1}{2} \dot{M}_{i} v^{2}
$$

is the energy flux vector. Eq. (7.12) constitutes the energy flux theorem. The energy flux may be written in a form related to a more familiar expression by expressing $\varphi$ in terms of the relative specific enthalpy $\bar{\epsilon}$. From (2.15) and (6.7) we derive

$$
\varphi=\Phi+\mathcal{G}=\bar{\epsilon}-T \bar{s}+\mathcal{G} .
$$

By introducing this value of $\varphi$ and the value (4.9) for $\dot{S}_{i}$ into expression (7.13) the energy flux becomes

$$
F_{i}=(\bar{\epsilon}+\mathcal{G}) \dot{M}_{i}+T \dot{S}_{i}{ }^{T}+\frac{1}{2} \dot{M}_{i} v^{2} .
$$

The term

$$
T \dot{S}_{i}^{T}=\dot{H}_{i}
$$

represents the heat flux by conduction in the compound fluid-solid medium.

In one-dimensional steady flow $\dot{M}_{i}=$ const., we neglect the conductive heat flux and 
the kinetic energy, and Eq. (7.12) reduces to

$$
\bar{\epsilon}+\mathcal{G}=\text { const. }
$$

For example, in a geothermal problem with vertical fluid flow it means that the relative specific enthalpy decreases by an amount equal to the increase of gravity potential $\mathcal{G}$. If $\mathcal{G}$ $=0$ the relative specific enthalpy $\bar{\epsilon}$ is constant. As a consequence we derive the well-known Joule-Thomson effect of vapor expansion through a porous plug.

8. Non-equilibrium adsorption. The foregoing results are applicable to the case of solid-fluid interaction represented by surface adsorption in the pores provided local equilibrium is assumed. However, the local equilibrium assumption is not valid with timedependent adsorption. The variational thermodynamic theory is readily generalized to such cases. We consider separately the mass displacement $M_{i}^{f}$ of the fluid in the pores and the mass displacement $M_{i}{ }^{a}$ in the adsorbed layer. We put

$$
m_{a}=\nu \xi-\frac{\partial M_{i}{ }^{a}}{\partial x_{i}} \quad, \quad m_{f}=-\nu \xi-\frac{\partial M_{i}{ }^{\prime}}{\partial x_{i}},
$$

where $m_{a}$ and $m_{f}$ are the masses adsorbed and pore fluid added per unit volume of porous medium. The quantity $\nu \xi$ represents the mass adsorbed from the fluid with a coefficient $\nu$ and a variable $\xi$ equal to zero in the initial state. The field variables are now $M_{i}{ }^{a}, M_{i}{ }^{f}, S_{i}, \xi$ and $s^{*}$. The collective entropy per unit volume is

$$
\mathcal{S}=-\frac{\partial S_{i}}{\partial x_{i}}+s^{*}
$$

where $s^{*}$ is the entropy produced and $S_{i}$ the total entropy displacement. The collective potential is

$$
V=V\left(m_{a}, m_{f}, \S\right)
$$

and

$$
d V=\Phi_{a} d m_{a}+\Phi_{f} d m_{f}+\theta d S .
$$

The convective potentials $\Phi_{a}$ and $\Phi_{f}$ are derived in exactly the same way as in Eq. (2.11) using corresponding thermobaric potentials. The rate of dissipation is

$$
T \dot{s}^{*}=2 D^{\prime}+\xi A\left(m_{a}, m_{f}, \delta\right)
$$

where

$$
D^{\prime}=\mathscr{D}^{\prime}\left(m_{a}, m_{f}, \mathcal{S}, \dot{M}_{i}{ }^{a}, \dot{M}_{i}{ }^{f}, \dot{S}_{i}\right)
$$

is a quadratic form in $\dot{M}_{i}{ }^{a}, \dot{M}_{i}{ }^{f}$ and $\dot{S}_{i}$ with coefficients functions of $m_{a}, m_{f}$ and $\delta$. The quantity $A$, also a function of $m_{a}, m_{f}$ and $\mathcal{S}$, plays the role of an affinity. If the medium is nonhomogeneous the values of $D^{\prime}$ and $A$ may also depend on the location $x_{i}$. An expression for $A$ is obtained by considering Eq. (2.3) with the value (8.4) for $d U$. We write

$$
\dot{\mathcal{U}}-T_{0} \dot{S}=\Phi_{a} \dot{m}_{a}+\Phi_{f} \dot{m}_{f}+\theta S .
$$

Consider a transformation such that $\dot{M}_{i}{ }^{a}=\dot{M}_{i}{ }^{f}=\dot{S}_{i}=0$. This implies $\dot{U}=0$ and $\dot{\mathcal{S}}=$ $\dot{s}^{*}$. With these values Eqs. (8.5) and (8.7) become

$$
T \dot{s}^{*}=A \dot{\xi}, T \dot{s}^{*}=\nu\left(\Phi_{f}-\Phi_{a}\right) \dot{\xi} .
$$


Hence

$$
A=\nu\left(\Phi_{f}-\Phi_{a}\right) .
$$

At equilibrium $A=0$ and $\Phi_{f}=\Phi_{a}$.

The rate equation for $\xi$ may be written in the form

$$
A=\xi K\left(m_{a}, m_{f}, \delta\right) .
$$

Hence (8.5) becomes

$$
T \dot{s}^{*}=2 D
$$

with a dissipation function

$$
D=D^{\prime}+\frac{1}{2} K \xi^{2} \text {. }
$$

We now impose arbitrary variations $\delta M_{i}{ }^{a}, \delta M_{i}{ }^{f}, \delta S_{i}$ and $\delta \xi$ on the field variables. Application of the principle of virtual dissipation, following exactly the same procedure as in Sec. 6 leads to the field equations

$$
\begin{array}{cl}
\frac{\partial \varphi_{a}}{\partial x_{i}}+\frac{\partial D}{\partial \dot{M}_{i}{ }^{a}}=0, & \frac{\partial \varphi_{f}}{\partial x_{i}}+\frac{\partial D}{\partial \dot{M}_{i}^{\prime}}=0, \\
\frac{\partial \theta}{\partial x_{i}}+\frac{\partial D}{\partial S_{i}}=0, & A+\frac{\partial D}{\partial \xi}=0,
\end{array}
$$

where $\varphi_{a}=\Phi_{a}+\mathcal{G}$ and $\varphi_{f}=\Phi_{f}+\mathcal{G}$. For simplicity we have neglected the accelerations.

In the more general context of irreversible thermodynamics the variable $\xi$ plays the role of a local internal coordinate, and the system exhibits viscoelastic properties discussed quite generally in earlier linear theories and more recently in the nonlinear case [3, 7]. The nature of the adsorption is quite general. It may include relaxation effects of fluid penetration in micropores.

9. Lagrangian equations and finite-element methods. The principle of virtual dissipation (5.12) leads directly to Lagrangian-type equations which provide a powerful tool for systems analysis in very complex technological problems. In particular, they are ideally suited for the treatment of geothermal systems. The method has been derived and applied repeatedly in earlier work [1, 2, 3]. More recently it was applied to the non-isothermal analysis of deformable porous media containing a fluid mixture [7]. As an illustration we shall apply the method to the case of a porous medium with non-equilibrium adsorption analyzed in sec. 8 . For simplicity we shall assume that the accelerations are negligible, and that the entropy produced $s^{*}$ may be neglected in the value (4.20) of $\delta$. The medium is then described by variables

$$
\begin{aligned}
{M_{i}}^{a} & =M_{i}{ }^{a}\left(q_{1} q_{2} \cdots q_{n} x_{l}\right), \\
M_{i}{ }^{f} & =M_{i}^{f}\left(q_{1} q_{2} \cdots q_{n} x_{l}\right), \\
S_{i} & =S_{i}\left(q_{1} q_{2} \cdots q_{n} x_{l}\right), \\
\xi & =\xi\left(q_{1} q_{2} \cdots q_{n} x_{l}\right),
\end{aligned}
$$

where $q_{i}$ are $n$ generalized coordinates. We write the principle of virtual dissipation (5.12), putting $I_{i}=\delta W^{\prime}=0$, as

$$
\delta P^{\prime}+\int_{\Omega} T \delta s^{*} d \Omega=0
$$


In the variation of $\delta \rho^{\prime}$ we have substituted the total variation $\delta$ instead of the restricted variation $\delta_{R}$ since $s^{*}$ is neglected in the value of $\delta$. Furthermore $P^{\prime}$ is defined as

$$
\delta \mathcal{P}^{\prime}=\delta \odot+\int_{A} \theta n_{i} \delta S_{i} d A+\int_{A} \varphi n_{i} \delta M_{i}{ }^{\prime} d A
$$

where

$$
\mathcal{P}=\int_{\Omega}\left[\mathcal{V}+\left(m_{0}+m_{a}+m_{f}\right) \mathcal{S}\right] d \Omega .
$$

The unit normal to the boundary $A$ of $\Omega$ is denoted by $n_{i}$.

The quantity $P^{\prime}$ represents the collective potential of the domain $\Omega$ plus an additional domain $C_{A}$ represented by driving cells distributed along the boundary $A$. This is done because application of the variational principle requires that no variations are applied at the boundary of the total domain $\Omega+C_{A}$. However, variations need not vanish at the boundary $A$ of $\Omega$. The virtual dissipation is derived from expressions (8.12) for D:

$$
\int_{\Omega} T \delta s^{*} d \Omega=\int_{\Omega}\left(\frac{\partial D}{\partial \dot{M}_{i}{ }^{a}} \delta M_{i}{ }^{a}+\frac{\partial D}{\partial \dot{M}_{i}{ }^{f}} \delta M_{i}{ }^{t}+\frac{\partial D}{\partial \dot{S}_{i}} \delta S_{i}+\frac{\partial D}{\partial \dot{\xi}} \delta \xi\right) d \Omega .
$$

This may be written

$$
\int_{\Omega} T \delta s^{*} d \Omega=\frac{\partial D}{\partial \dot{q}_{i}} \delta q_{i}
$$

with the total dissipation function

$$
D=\int_{\Omega} D d \Omega=\frac{1}{2} b_{i j}\left(q_{l}\right) \dot{q}_{i} \dot{q}_{j}
$$

It is a quadratic form in $\dot{q}_{i}$ with coefficients functions of $q_{i}$. Substitution of (9.3) and (9.6) into the variational principle (9.2) and cancellation of the coefficients of $\delta q_{i}$ yields the $n$ Langrangian equations

$$
\frac{\partial P}{\partial q_{i}}+\frac{\partial D}{\partial \dot{q}_{i}}=Q_{i}
$$

where

$$
Q_{i}=-\int_{A}\left(\theta n_{j} \frac{\partial S_{j}}{\partial q_{i}}+\varphi n_{j} \frac{\partial M_{j}^{f}}{\partial q_{i}}\right) d A
$$

is the generalized thermodynamic driving force.

These equations are the same as those obtained in heat transfer problems. Numerous techniques for the use of these equations have been developed [9] and are applicable here.

Finite-element method. The Lagrangian equations (9.8) yield immediately a large variety of finite-element methods. For example, we may divide the domain $\Omega$ into tetrahedral elements whose vertices constitute the nodes of the network. Values of the variables $M_{i}{ }^{a} M_{i}{ }^{f} S_{i}$ and $\xi$ at the nodes may be chosen as generalized coordinates $q_{i}$ while their values inside tetrahedral cells are determined by linear interpolation of the values $q_{i}$.

If needed, the value of $s^{*}$ may be taken into account, as already suggested in [7], by introducing the values of $s^{*}$ at the vertices as additional unknowns and using linear interpolation for the value $s^{*}$ inside the cells. The required additional equations are obtained by verifying Eq. (8.11) at each node. 


\section{REFERENCES}

[1] M. A. Biot, A virtual dissipation principle and Lagrangian equations in nonlinear irreversible thermodynamics, Bull. Roy. Acad. Belgium (Classes des Sciences) 61, 6-30 (1975)

[2] M. A. Biot, Variational principles in irreversible thermodynamics with application to viscoelasticity, Phys. Rev. 97, 1463-1469 (1955)

[3] M. A. Biot, Variational-Lagrangian irreversible thermodynamics of nonlinear thermorheology, Quart. Appl. Math. 24, 213-248 (1976)

[4] M. A. Biot, New chemical thermodynamics of open systems. Thermobaric potential, a new concept, Bull. Roy. Acad. Belgium (Classe des Sciences) 62, 239-258 (and Erratum p. 678) (1976)

[5] M. A. Biot, New fundamental concepts and results in thermodynamics with chemical applications, Chemical Physics 22, 183-198 (1977)

[6] M. A. Biot, Variational thermodynamics of viscous compressible heat-conducting fluids, Quart. Appl. Math. 34, 323-329 (1977)

[7] M. A. Biot, Variational-Lagrangian thermodynamics of nonisothermal finite strain mechanics of porous solids and thermomolecular diffusion, Int. J. Solids Structures 13, 579-597 (1977)

[8] J. Willard Gibbs, Scientific papers, Vol. 1. Thermodynamics, Longmans Green and Co., London, 1906

[9] M. A. Biot, Variational principles in heat transfer. A unified Lagrangian analysis of dissipative phenomena. Oxford Press, London, 1970

[10] J. Meixner, Zur Thermodynamik der Thermodiffusion, Ann. Physik 39, 333-356 (1941)

[11] L. Onsager, Reciprocal relations in irreversible processes I, Phys. Rev. 37, 405-426 (1930)

[12] L. Onsager, Reciprocal relations in irreversible thermodynamics 1I. Phys. Rev. 37, 2265-2279 (1931)

[13] S. R. De Groot, Thermodynamics of irreversible processes, North Holland Publishing Co., Amsterdam, 1952

[14] M. King Hubbert, The theory of ground-water motion, J. Geology, 48, 785-944 (1940)

[15] A. E. Scheidegger, The physics of flow through porous media, Macmillan, New York, 1960 